\title{
Artificial intelligence for the general cardiologist
}

\author{
J. Verjans · T. Leiner
}

Published online: 21 August 2019

(c) The Author(s) 2019

The majority of experts and opinion leaders believe that artificial intelligence (AI) is going to revolutionise many industries, including healthcare [1]. In the short term, the power and potential of AI appear most suitable for complementing human expertise. In other words, machines will help humans do a better job. Consequently, it is anticipated that AI will help with repetitive tasks, in-depth quantification and classification of findings, improved patient and disease phenotyping and, ultimately, with better outcomes for patients, physicians, hospital administrators, insurance companies and governments [2].

Despite these promises, the impact of AI in current clinical practice is still limited. However, this could change in the coming years, as illustrated by the significant increase in papers in AI, machine learning and deep learning in cardiology (Fig. 1). Moreover, multiple applications have gained Federal Drug Administration approval in recent years with significant financial support; these are directly related to daily cardiology practice, including automated interpretation of electrocardiograms, automated segmentation and diagnosis (Tab. 1). Common practice in invasive cardiology will be seriously influenced by AI, for example by predicting the outcome of interventions such as transcatheter aortic valve implantation or AI-based

\section{J. Verjans $(\triangle)$}

Royal Adelaide Hospital, Adelaide, SA, Australia

South Australian Health and Medical Research Institute, Adelaide, SA, Australia

Australian Institute for Machine Learning, University of Adelaide, Adelaide, SA, Australia

Johan.Verjans@SAHMRI.com

\section{T. Leiner}

Department of Radiology, University Medical Centre

Utrecht, Utrecht University, Utrecht, The Netherlands non-invasive estimation of the haemodynamic significance (CT fractional flow reserve) of coronary artery stenosis on CT angiography that is being developed by different companies [3-5].

This focus issue of the Netherlands Heart Journal aims to help general cardiologists explore the state of the art of AI in cardiology. It also aims to increase awareness that it is likely that AI is going to influence and even disrupt daily clinical practice and healthcare in general.

The first part of the issue will focus on past, present and future evidence and gives an overview in the form of two reviews on the impact of AI in cardiology and an area of more immediate impact, cardiovascular imaging $[6,7]$. In the second part of this issue, several Dutch flagship AI projects are discussed and demonstrate the potential in various areas of AI as discussed above. These efforts not only concern clinical practical problems such as cardiovascular imaging and risk assessment, but also the need for multidisciplinary collaboration [8] and dedicated data platforms to access and analyse the collected data $[9,10]$.

According to scientists from every decade since the 1960s, human-like AI should have been achieved within 10-20 years. However, mainly due to a lack of computational power, there have been two so-called 'winters' for AI around 1980 and 1993, both after a period of increased interest. But with the recent increase in computational power, smarter use of hardware and data, and new strategies such as deep learning, this appears to have changed for good. Artificial intelligence is likely to make its potential come true in the era of complex data, serving as a conduit to insight for doctors in their patient's data, improving efficiency and reducing errors.

Let's plan some randomised clinical trials! 


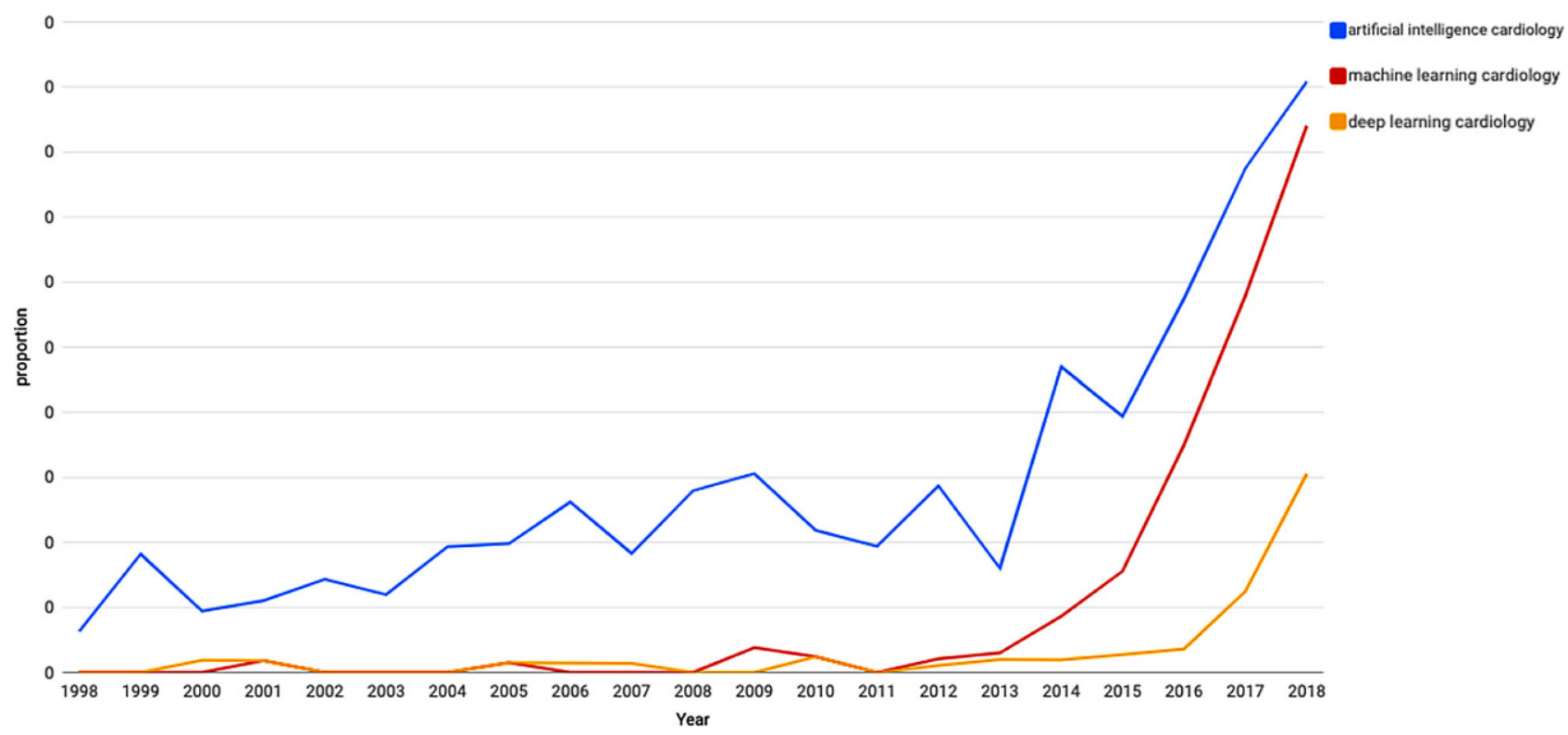

Fig. 1 Proportion of citations on PubMed (1998-2018) for each search per year for key terms (source: http://esoerr.github.io/ pubmed-by-year)

Table 1 Federal Drug Administration approvals of artificial intelligence $(A I)$ applications relevant for cardiology practice

\begin{tabular}{|l|l|l|}
\hline Year & Company & Al application \\
\hline 2014 & Alivecor & Detection of atrial fibrillation \\
\hline 2016 & Lumify & Ultrasound image diagnosis \\
\hline 2017 & Arterys & Cardiac MRI ventricle segmentation \\
\hline 2017 & Bioflux & Detecting arrhythmias \\
\hline 2017 & Cardiologs & ECG analysis \\
\hline 2018 & Lepu Medical & ECG analysis \\
\hline 2018 & Zebra Medical Vision & Automated coronary artery calcification \\
\hline 2018 & $\begin{array}{l}\text { Physiq Heart Rhythm } \\
\text { Module }\end{array}$ & Detection of atrial fibrillation \\
\hline 2018 & Apple & Detection of atrial fibrillation \\
\hline 2018 & Bay Labs & Echocardiogram analysis \\
\hline 2019 & Verily & ECG feature on Study Watch \\
\hline 2019 & Alivecor & Six-lead smartphone ECG \\
\hline 2019 & Zebra Medical Vision & Radiographic diagnosis \\
\hline 2019 & Aidoc & Flagging pulmonary embolism \\
\hline
\end{tabular}

Conflict of interest J. Verjans declares that he has no competing interests. T. Leiner declares: Activities related to the present article: disclosed no relevant relationships. Activities not related to the present article: disclosed institutional grant received from Pie Medical B.V.; payments received from Bayer Healthcare and Philips Healthcare for lectures including service on speakers' bureaus. Other relationships: disclosed that he is coinventor of U.S. patent 10176575 ; this patent is held by Utrecht University Holdings, which manages the terms of any licensing agreement.

Open Access This article is distributed under the terms of the Creative Commons Attribution 4.0 International License (http://creativecommons.org/licenses/by/4.0/), which permits unrestricted use, distribution, and reproduction in any medium, provided you give appropriate credit to the origi- nal author(s) and the source, provide a link to the Creative Commons license, and indicate if changes were made.

\section{References}

1. The return of the machinery question. Economist. 25 June. 2016. http://www.economist.com/news/special-report/ 21700761-after-many-false-starts-artificial-intelligencehas-taken-will-it-cause-mass. Accessed 1 Aug 2019.

2. Rajkomar A, Dean J, Kohane I, et al. Machine learning in medicine. NEngl J Med. 2019;380:1347-58.

3. Coenen A, Kim YH, Kruk M, et al. Diagnostic accuracy of a machine-learning approach to coronary computed tomographic angiography-based fractional flow reserve: result from the MACHINE Consortium. Circ Cardiovasc Imaging. 2018;11:e7217. Jun.

4. Douglas PS, Pontone G, Hlatky MA, et al. Clinical outcomes of fractional flow reserve by computed tomographic angiography-guided diagnostic strategies vs. usual care in patients with suspected coronary artery disease: the prospective longitudinal trial of FFR(CT): outcome and resource impacts study. Eur Heart J. 2015;14;36:3359-67. Dec.

5. Benjamins JW, Hendriks T, Knuuti J, et al. A primer in artificial intelligence in cardiovascular medicine. Neth Heart J. 2019;27. https://doi.org/10.1007/s12471-019-1286-6.

6. Siegersma KR, Leiner T, ChewDP, et al. Artificial intelligence in cardiovascularimaging - state of the artandimplications for the imaging cardiologist. Neth Heart J. 2019;27. https:// doi.org/10.1007/s12471-019-01311-1.

7. Benjamins JW, van Leeuwen K, Hofstra L, et al. Enhancing cardiovascular artificial intelligence (AI) research in the Netherlands: CVON-AI consortium. Neth Heart J. 2019;27. https://doi.org/10.1007/s12471-019-1281-y.

8. Sammani A, Jansen M, Linschoten M, et al. UNRAVEL: big data analytics research data platform to improve care of patients with cardiomyopathies using routine electronic health records and standardised biobanking. Neth Heart J. 2019;27. https://doi.org/10.1007/s12471-019-1288-4. 
9. Groenhof TKJ, Rittersma ZH, Bots ML, et al. A computerised decision support system for cardiovascular risk management 'live' in the electronic health record environment: development, validation and implementation: the.
Utrecht: Cardiovascular Cohort Initiative. Neth Heart J. 2019;27. https://doi.org/10.1007/s12471-019-01308-w.

10. Lopes RR, van Mourik M, Schaft EV, et al. Value of machine learningin predictingTAVIoutcomes. Neth HeartJ. 2019;27. https://doi.org/10.1007/s12471-019-1285-7. 